\title{
Eyelash trichomegaly following treatment with erlotinib in a non-small cell lung cancer patient: A case report and literature review
}

\author{
SHAN-BING WANG ${ }^{1}$, KAI-JIAN LEI ${ }^{1}$, JIA-PEI LIU ${ }^{2}$ and YU-MING JIA ${ }^{1}$ \\ Departments of ${ }^{1}$ Oncology and ${ }^{2}$ Laboratory Medicine, \\ The Second People's Hospital of Yibin, Yibin, Sichuan 644000, P.R. China
}

Received August 31, 2014; Accepted April 4, 2015

DOI: $10.3892 / 01.2015 .3265$

\begin{abstract}
Inhibitors of epidermal growth factor receptor (EGFR), including tyrosine kinase inhibitors (TKIs), present significant clinical benefits in the treatment of non-small cell lung cancer (NSCLC), particularly in patients with an EGFR mutation. However, TKI treatment also results in unwanted cutaneous toxic side effects, such as a skin rash. Eyelash trichomegaly is rarely reported as a side effect; however, it causes cosmetic issues or eyeball irritation in patients, which may result in the early termination of TKI treatment. Therefore, although TKI-induced eyelash trichomegaly is rare, it should be considered carefully by lung cancer physicians. The present study reported a case of erlotinib-induced eyelash trichomegaly in a 65-year-old Chinese female patient suffering from NSCLC with an EGFR mutation. To the best of our knowledge, this is the first reported case of erlotinib-induced trichomegaly in a Chinese patient.
\end{abstract}

\section{Introduction}

Lung cancer is a leading cause of cancer-associated mortality worldwide (1). Targeted therapy has been developed and is widely used for the treatment of non-small cell lung cancer (NSCLC), particularly in patients harboring an activating epidermal growth factor receptor (EGFR) mutation (2). The IRESSA Pan-Asia Study trial found that patients with sensitizing EGFR mutations who received gefitinib had increased progression-free survival (24.9\%) when compared with patients treated with carboplatin-paclitaxel $(6.7 \%)$, as well as an increased response rate (71.2 vs. 47.3\%) (3). Based on these results, the Food and Drug Administration (FDA)

Correspondence to: Dr Shan-Bing Wang, Department of Oncology, The Second People's Hospital of Yibin, 97 Beida Street, Yibin, Sichuan 644000, P.R. China

E-mail: 275915691@qq.com

Key words: eyelash trichomegaly, epidermal growth factor receptor, tyrosine kinase inhibitor, non-small cell lung cancer, erlotinib approved erlotinib for the treatment of patients with locally advanced or metastatic NSCLC after progression on at least one prior chemotherapy regimen. With the wide use of EGFR tyrosine kinase inhibitors (TKIs) in patients with NSCLC, an increasing number of side effects have been observed, including skin rash, diarrhea, stomatitis and eyelash trichomegaly $(4,5)$. The adverse events most frequently reported by erlotinib-treated patients are rash $(56.0 \%)$ and diarrhea $(62.0 \%)(6)$. Although erlotinib exhibits a number of side effects, it presents significant clinical benefits in the treatment of non-small cell lung cancer (NSCLC), particularly in patients with an EGFR mutation (6). Recently, the FDA approved the use of erlotinib as first-line therapy in patients with exon 19 deletions or exon 21 (L858R) substitution mutations (6). Eyelash trichomegaly, which was first identified by Gray et al (7) in 1944, is characterized by the increased length, thickness, stiffness, curling and pigmentation of the eyelashes. EGFR TKI-associated eyelash trichomegaly has been rarely reported and its incidence remains unknown (4). In this study, a Chinese female patient with NSCLC that developed eyelash trichomegaly following the administration of erlotinib is presented. To the best our knowledge, this is the first case of trichomegaly associated with erlotinib treatment to be reported in the literature. As lung cancer physicians administer EGFR TKIs with increasing frequency, this untoward effect requires attention.

\section{Case report}

In May 2011, a 65-year-old Chinese female with no history of smoking presented at the Second People's Hospital of Yibin (Yibin, China) with isolated coughing that had persisted for several months. A chest computed tomography (CT) scan revealed a $2.8 \times 3 \mathrm{~cm}$ mass on the upper lobe of the right lung and widespread bilateral pulmonary nodules consistent with metastases (Fig. 1A and B). A bone scan revealed the presence of multiple metastases (pelvis and spine). However, no evidence of metastases was detected in a brain magnetic resonance imaging scan. Subsequently, a CT-guided fine-needle aspirate biopsy of the lung lesion was performed. Immunohistology revealed that the tumor cells were positive for cytokeratin 7 , thyroid transcription factor 1 , 

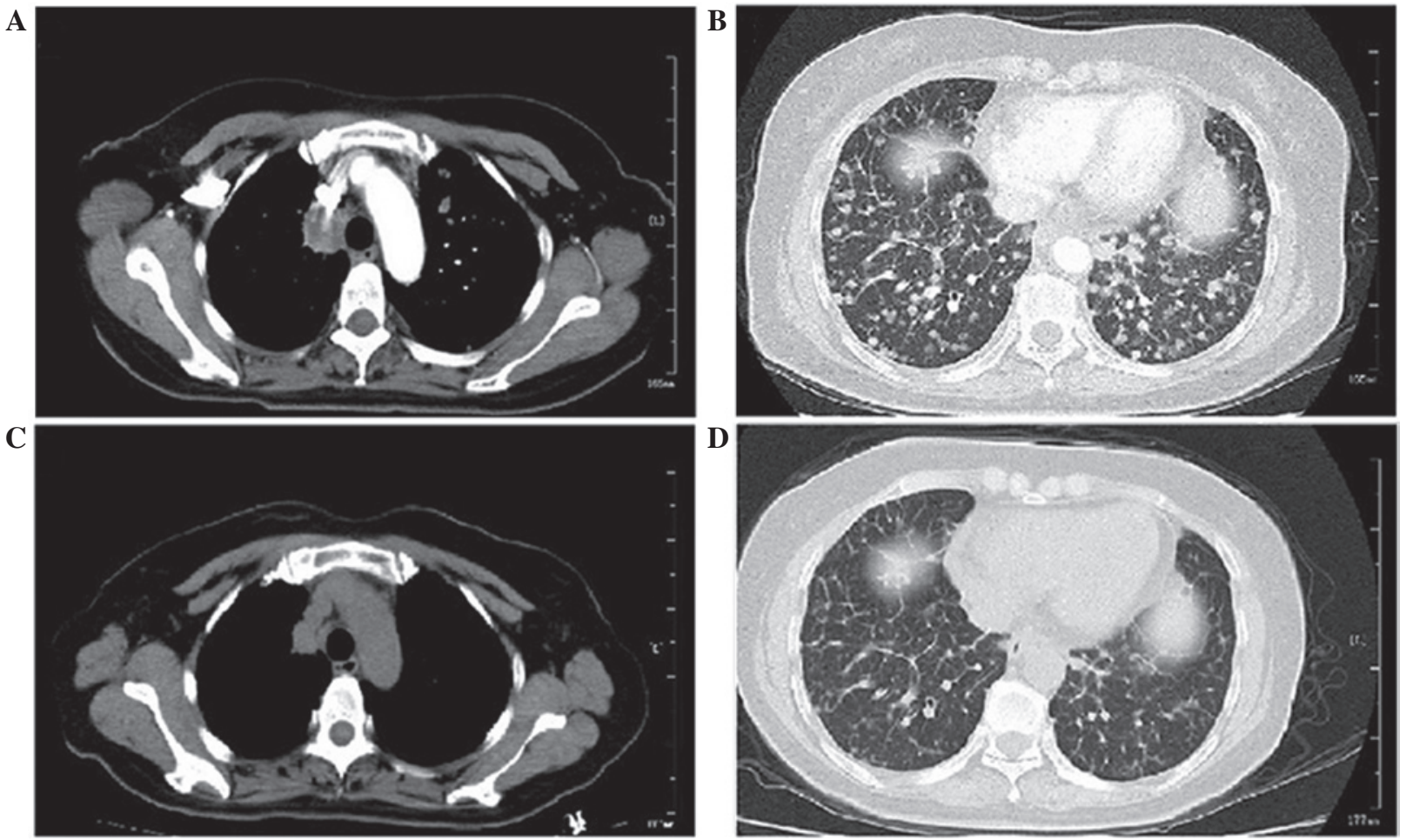

Figure 1. (A and B) Enhanced chest CT scans prior to the treatment, revealing a mass on the upper lobe of the right lung and widespread bilateral pulmonary nodules. (C and D) Unenhanced chest CT scans after 1 month of treatment with erlotinib, demonstrating partial response to the treatment. CT, computed tomography.

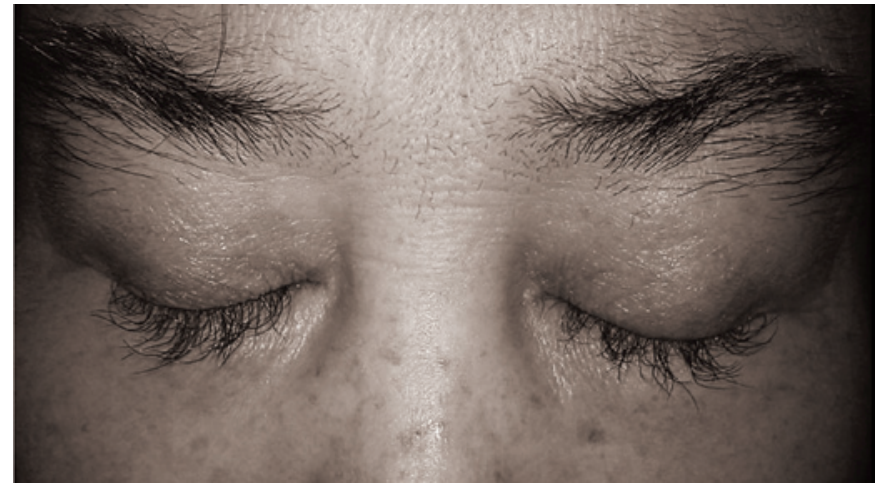

Figure 2. Trichomegaly of eyelashes developed within 5 months of erlotinib treatment.

NapsinA and EGFR, and a diagnosis of adenocarcinoma was determined. Polymerase chain reaction-based DNA sequencing of the $E G F R$ gene revealed an activating mutation (L858R) in exon 21 of the EGFR gene. The patient declined to undergo systemic chemotherapy treatment due to concerns regarding possible side effects. In May 2011, the patient was administered erlotinib monotherapy, at a single daily dose of $150 \mathrm{mg}$. Within 4 weeks of treatment, a significant symptomatic improvement in exercise tolerance was observed and almost complete resolution of coughing. Radiological imaging demonstrated a partial response to the treatment (Fig. 1C and D). During the 6-month erlotinib treatment, the patient experienced a moderate rash on her face, chest and back, which was treated with topical clindamycin. In addition, after 5 months of treatment, the patient's eyelashes were overgrowing, showing increased length and thickness (trichomegaly) which caused visual disturbance, and necessitated trimming (Fig. 2).

Despite the initial response to erlotinib, an assessment performed in November 2011 revealed progression of the cancer and, after approximately 2 weeks, the patient succumbed to the disease.

The current study was approved by the Ethics Committee of The Second People's Hospital of Yibin. Written informed consent was obtained from the patient's family.

\section{Discussion}

Eyelash trichomegaly is defined as the increased length, thickness, stiffness, curling and pigmentation of the eyelashes (7). This condition was initially identified in patients with certain congenital syndromes, including the Oliver-McFarlane syndrome (8), Cornelia de Lange syndrome (9) or familial hypertrichosis (10). Acquired trichomegaly is also associated with specific drugs $(11,12)$ and other diseases, including vernal keratoconjunctivitis and atopic dermatitis $(13,14)$. However, eyelash trichomegaly is not a drug-limiting side effect.

Recently, with the wide use of erlotinib in NSCLC patients, an increased number of cutaneous adverse effects, including acneiform rash or pruritus, have been observed (6). Erlotinib-associated eyelash trichomegaly has been reported only in a small number of case reports $(4,5,15-17)$. To the best of our knowledge, erlotinib-induced trichomegaly has not been previously reported in Chinese patients. Additionally, the pathogenesis of these symptoms associated with the administration of erlotinib is largely unknown. A previous study 
proposed that systemic inhibition of EGFRs with erlotinib not only affects the apoptosis and proliferation of cancerous cells, but is also able to affect the progression of hair follicles from the anagen to the telogen phase (18). This leads to an aberrant anagen phase and subsequently to abnormal hair growth (18), which can stimulate the formation of a disorganized hair follicles.

The EGFR, a transmembrane glycoprotein, is a member of the tyrosine kinase growth factor receptor family (19) and is expressed in the vast majority of patients with NSCLC. The most common EGFR mutations in patients with NSCLC include a deletion in exon 19 (E19del) and a mutation in exon 21 (L858R) (20); these two mutations are predictive of the NSCLC patient response and survival following EGFR TKI treatment $(21,22)$. A prospective head-to-head phase 3 study has demonstrated an overall response rate of $83 \%$ with a median progression-free survival of 13.1 months following first-line erlotinib therapy in Chinese patients with advanced NSCLC, who have tumor harboring an activating EGFR mutation (exon 19 deletion, 52\%; L858R mutation, 48\%) (23). In addition, other factors, such as the cutaneous rash severity, have been established as positive predictive markers of the clinical benefits following treatment (24). Similarly, the patient of the current study presented an activating mutation (L858R) in exon 21 and experienced a moderate rash on her face, chest and back; however, the patient did not present similar progression-free survival benefits. Considering the present case along with similar cases previously reported in the literature $(5,16,25)$ led to several interesting observations. Patients who manifested trichomegaly also exhibited a poor progression-free survival (3-7 months), and disease progression occurred after 1-3 months of trichomegaly. Therefore, eyelash trichomegaly may correlate with the resistance of lung cancer to EGFR inhibitors.

Although trichomegaly is not a drug-limiting side effect, it can obscure vision and has been reported to cause corneal problems, including erosions $(26)$, irritation $(15,26)$, infection and ulcers (17). Trimming and epilation of the elongated eyelashes are the most common and safe therapeutic options used by patients experiencing drug-associated eyelash trichomegaly $(4,15,17,26)$. In conclusion, trichomegaly is a rare, EGFR TKI-associated effect. Oncologists should be aware of this potential sequela, for which referral to an ophthalmologist or dermatologist may be helpful.

\section{References}

1. Ferlay J, Shin HR, Bray F, Forman D, Mathers C and Parkin DM: Estimates of worldwide burden of cancer in 2008: GLOBOCAN 2008. Int J Cancer 127: 2893-2917, 2010.

2. Shepherd FA, Rodrigues Pereira J, Ciuleanu T, et al; National Cancer Institute of Canada Clinical Trials Group: Erlotinib in previously treated non-small-cell lung cancer. N Engl J Med 353: 123-132, 2005.

3. Mok TS, Wu YL, Thongprasert S, et al: Gefitinib or carboplatin-paclitaxel in pulmonary adenocarcinoma. N Engl J Med 361: 947-957, 2009.

4. Braiteh F, Kurzrock R and Johnson FM: Trichomegaly of the eyelashes after lung cancer treatment with the epidermal growth factor receptor inhibitor erlotinib. J Clin Oncol 26: 3460-3462, 2008.
5. Jeon SH, Ryu JS, Choi GS, et al: Erlotinib induced trichomegaly of the eyelashes. Tuberc Respir Dis (Seoul) 74: 37-40, 2013.

6. Khozin S, Blumenthal GM, Jiang X, et al: U.S. Food and Drug Administration approval summary: Erlotinib for the first-line treatment of metastatic non-small cell lung cancer with epidermal growth factor receptor exon 19 deletions or exon 21 (L858R) substitution mutations. Oncologist 19: 774-779, 2014.

7. Gray H: Trichomegaly or movie lashes. Stanford Med Bull 2: 157-158, 1944.

8. Oliver GL and McFarlane DC: Congenital trichomegaly: With associated pigmentary degeneration of the retina, dwarfism, and mental retardation. Arch Ophthalmol 74: 169-171, 1965.

9. Kline AD, Krantz ID, Sommer A, et al: Cornelia de Lange syndrome: Clinical review, diagnostic and scoring systems and anticipatory guidance. Am J Med Genet A 143A: 1287-1296, 2007.

10. Ziakas NG, Jogiya A and Michaelides M: A case of familial trichomegaly in association with oculocutaneous albinism type 1. Eye (Lond) 18: 863-864, 2004.

11. Cohen PR, Escudier SM and Kurzrock R: Cetuximab-associated elongation of the eyelashes: Case report and review of eyelash trichomegaly secondary to epidermal growth factor receptor inhibitors. Am J Clin Dermatol 12: 63-67, 2011.

12. Pascual JC, Bañuls J, Belinchon I, Blanes M and Massuti B: Trichomegaly following treatment with gefitinib (ZD1839). Br J Dermatol 151: 1111-1112, 2004.

13. Pucci N, Novembre E, Lombardi E, et al: Long eyelashes in a case series of 93 children with vernal keratoconjunctivitis. Pediatrics 115: e86-e91, 2005.

14. Alpsoy E: Hypotrichosis, long eyelashes and atopic dermatitis: A new syndrome? J Eur Acad Dermatol Venereol 18: 374-375, 2004.

15. Carser JE and Summers YJ: Trichomegaly of the eyelashes after treatment with erlotinib in non-small cell lung cancer. J Thorac Oncol 1: 1040-1041, 2006.

16. Iacovelli R, Palazzo A, Trenta P, et al: Trichomegaly of the eyelashe induced by erlotinib therapy. Lung Cancer 64 (Suppl 1): S59, 2009.

17. Lane K and Goldstein SM: Erlotinib-associated trichomegaly. Ophthal Plast Reconstr Surg 23: 65-66, 2007.

18. Vergou T, Stratigos AJ, Karapanagiotou EM, et al: Facial hypertrichosis and trichomegaly developing in patients treated with the epidermal growth factor receptor inhibitor erlotinib. J Am Acad Dermatol 63: e56-e58, 2010.

19. Yarden Y and Sliwkowski MX: Untangling the ErbB signalling network. Nat Rev Mol Cell Biol 2: 127-137, 2001.

20. Hirsch FR and Bunn PA Jr: EGFR testing in lung cancer is ready for prime time. Lancet Oncol 10: 432-433, 2009.

21. Miller VA, Riely GJ, Zakowski MF, et al: Molecular characteristics of bronchioloalveolar carcinoma and adenocarcinoma, bronchioloalveolar carcinoma subtype, predict response to erlotinib. J Clin Oncol 26: 1472-1478, 2008.

22. Sequist LV, Martins RG, Spigel D, et al: First-line gefitinib in patients with advanced non-small-cell lung cancer harboring somatic EGFR mutations. J Clin Oncol 26: 2442-2449, 2008.

23. Zhou C, Wu YL, Chen G, et al: Erlotinib versus chemotherapy as first-line treatment for patients with advanced EGFR mutation-positive non-small-cell lung cancer (OPTIMAL, CTONG-0802): A multicentre, open-label, randomised, phase 3 study. Lancet Oncol 12: 735-742, 2011.

24. Petrelli F, Borgonovo K, Cabiddu M, Lonati V and Barni S: Relationship between skin rash and outcome in non-small-cell lung cancer patients treated with anti-EGFR tyrosine kinase inhibitors: A literature-based meta-analysis of 24 trials. Lung Cancer 78: 8-15, 2012.

25. Bouché O, Brixi-Benmansour H, Bertin A, Perceau G and Lagarde S: Trichomegaly of the eyelashes following treatment with cetuximab. Ann Oncol 16: 1711-1712, 2005.

26. Shah NT, Kris MG, Pao W, et al: Practical management of patients with non-small-cell lung cancer treated with gefitinib. J Clin Oncol 23: 165-174, 2005. 